\title{
Kinetics Study of a Complex Reaction: Nitration of Caged 2,6,8,12-Tetraacetyl-4,10-dinitro-2,4,6,8,10,12- hexaazaisowurtzitane
}

Yiying Zhang, ${ }^{\dagger}$ Dongxiang Zhang, ${ }^{\dagger}$ Kai Dong, ${ }^{\dagger}$ Penghao Lv, ${ }^{\dagger}$ Siping Pang, ${ }^{\dagger}$ and Chenghui Sun ${ }^{*}, \dagger$

${ }^{\dagger}$ School of Materials Science \& Engineering, Beijing Institute of Technology, 5 South Zhongguancun Street, Haidian District, Beijing 100081, P.R. China

${ }^{\ddagger}$ School of Chemical Engineering and Environment, Beijing Institute of Technology, 5 South Zhongguancun Street, Haidian District, Beijing 100081, P.R. China

*Corresponding author: Tel.: (+)86 010-68918049. Fax: (+)86 010-68918049.

E-mail:sunch@ bit.edu.cn.

\section{Supporting Information}

S1 Naming rule of hexaazaisowurtzitane derivatives

S2 HPLC-MS method for analysis of the reaction samples

S3 MS identification of nitration intermediates

S4 Quantitative analysis method of reaction components

S5 Calculation formula of $R, R^{2}$ and adjusted $R^{2}$ 


\section{S1. Naming rule of hexaazaisowurtzitane derivatives}

HNIW and its various precursors are derived from an idential fundational structure-a hexaazaisowurtzitane cage molecule, which consists of one six-membered nitrogen containing heterocycle and two five-membered nitrogen containing heterocycles. The six-membered hetercycle makes up the bottom of the cage and the two five-membered hetercycles linked by a C-C bond lie above it. The twelve $\mathrm{C}$ and $\mathrm{N}$ atoms in the cage skeleton are conventionally numbered as shown in Figure S1. Various hexaazaisowurtzitane derivatives can be obtained by introducing different substituent groups onto the $\mathrm{N}$ atoms and they are named according to the kinds and positions of the substituent groups.

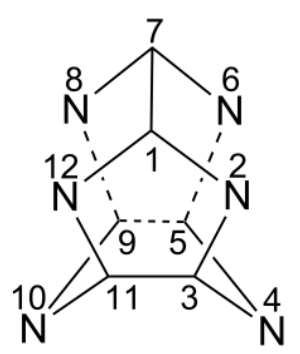

Figure S1. The molecule structure of hexaazaisowurtzitane cage.

Four nitration intermediates are taken as examples to illustrate the naming rule as follows.

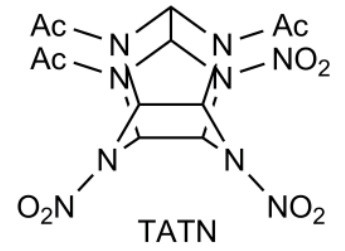

6,8,12-triacetyl-2,4,10-trinitro-

2,4,6,8,10,12-hexaazaisowurtzitan

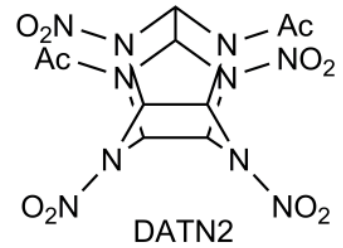

6,12-diacetyl-2,4,8,10-tetranitro-

2,4,6,8,10,12-hexaazaisowurtzitane

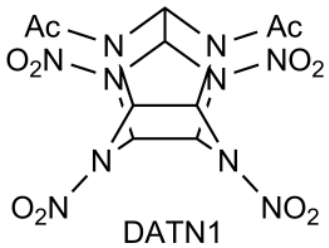

6,8-diacetyl-2,4,10,12-tetranitro-

2,4,6,8,10,12-hexaazaisowurtzitane

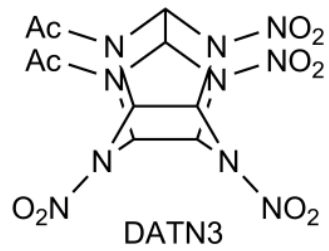

8,12-diacetyl-2,4,6,10-tetranitro-

2,4,6,8,10,12-hexaazaisowurtzitane

Figure S2. Some hexaazaisowurtzitane derivatives and their names. 


\section{S2. HPLC-MS method for analysis of the reaction samples}

HPLC analysis conditions:

Column: Eclipse XDB C18 (5 um, $4.6 \mathrm{~mm} \times 250 \mathrm{~mm})$

Solvent A: water $60 \%$

Solvent B: methanol 40\%

Sample injection: 5 ul

Wavelength: $230 \mathrm{~nm}$

Flow rate: $0.4 \mathrm{~mL} / \mathrm{min}$

The retention time of every reaction component under the analysis condition are shown in

Table S1:

Table S1. The retention time of reaction components

\begin{tabular}{|c|c|c|c|c|c|c|c|c|}
\hline Components & TADN & TATN & DATN1 & DATN2 & DATN3 & MPIW & HNIW & Acetic \\
\hline Retention & 8.15 & 10.73 & 12.23 & 14.52 & 18.79 & 22.84 & 42.61 & 6.18 \\
time $(\min )$ & & & & & & & & \\
\hline
\end{tabular}

MS Conditions:

Ion polarity: Negative; $\quad$ Ion source Type: ESI;

Dry temp: $250{ }^{\circ} \mathrm{C} ; \quad$ Dry gas: $12 \mathrm{~mL} / \mathrm{min}$;

Capillary voltage: $4000 \mathrm{~V} ; \quad$ Nebulizer: 35 psig; Frag: 70. 
S3. MS identification of nitration intermediates

TADN (M=426)

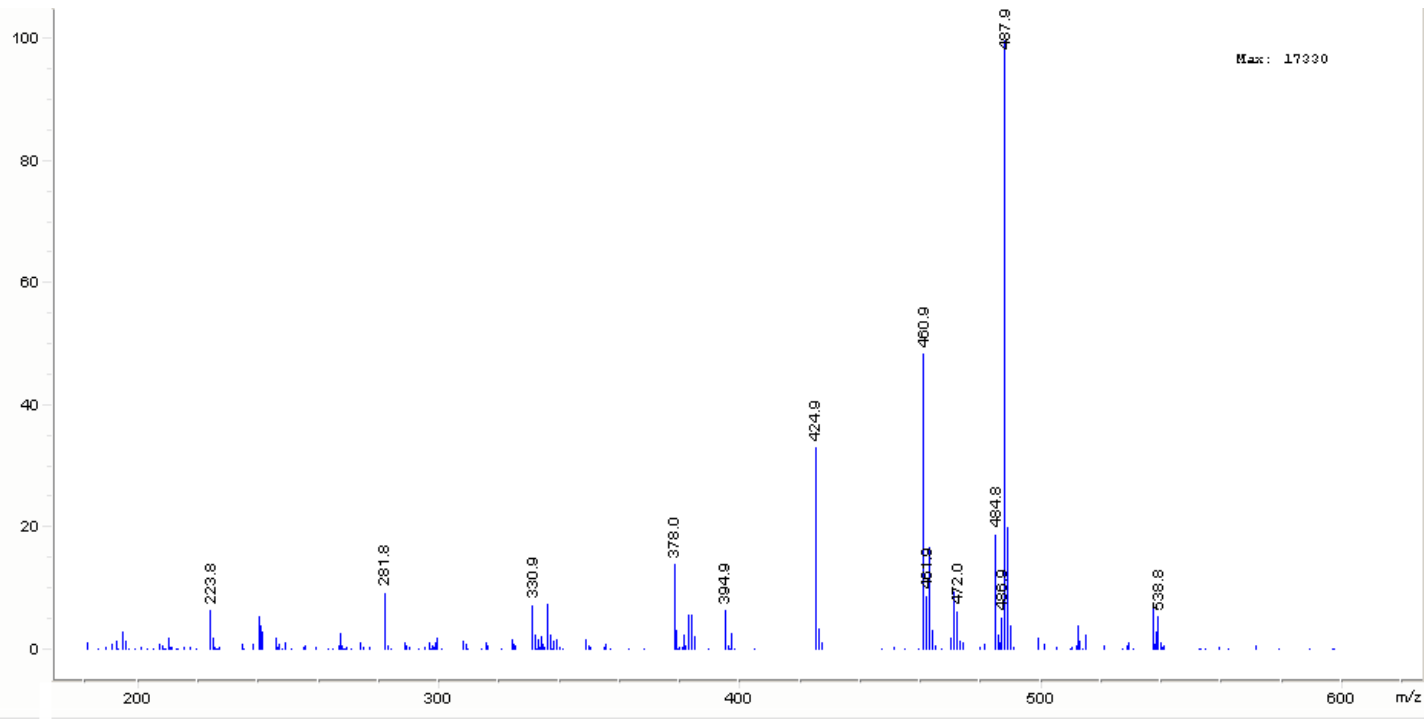

Figure S3. MS spectrum of TADN

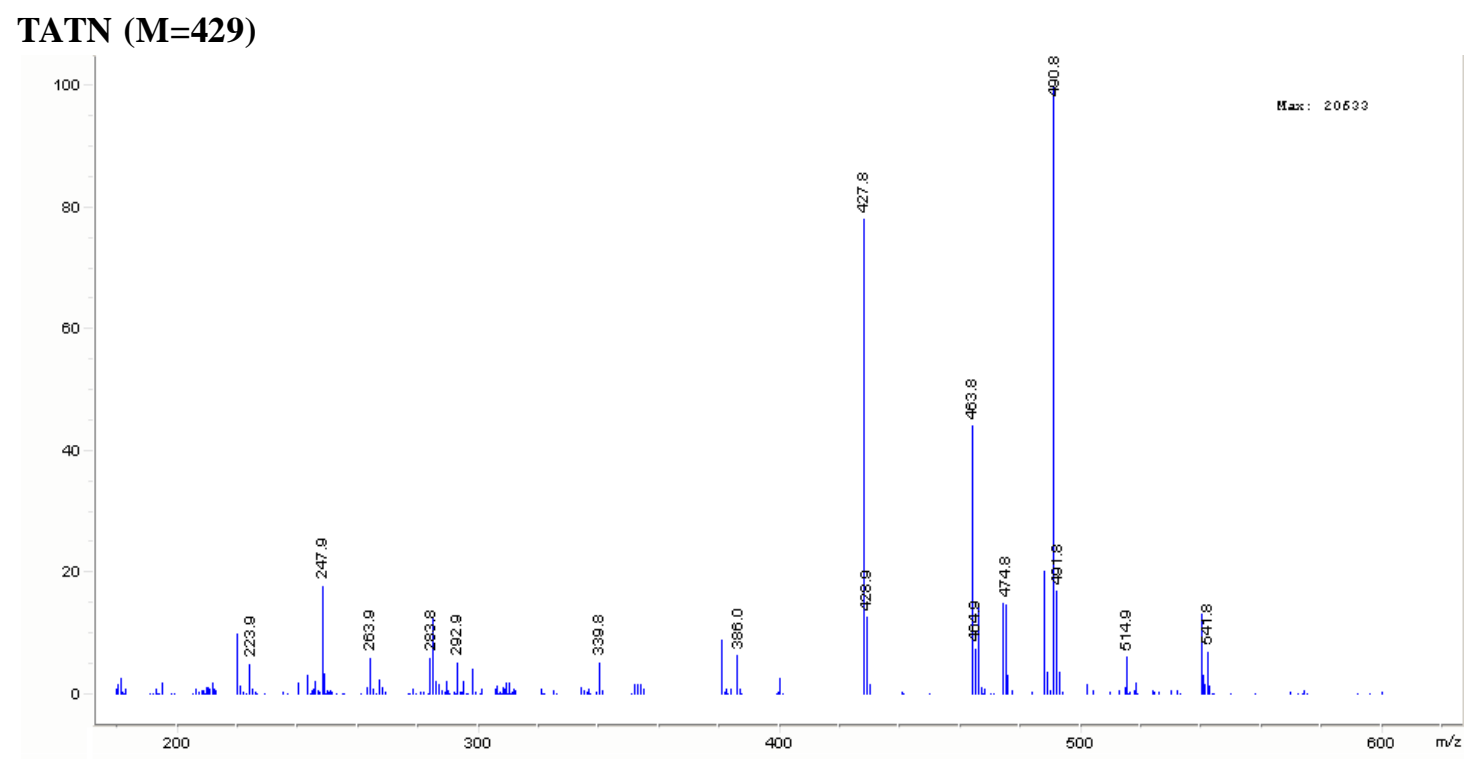

Figure S4. MS spectrum of TATN 


\section{DATN1 $(M=432)$}

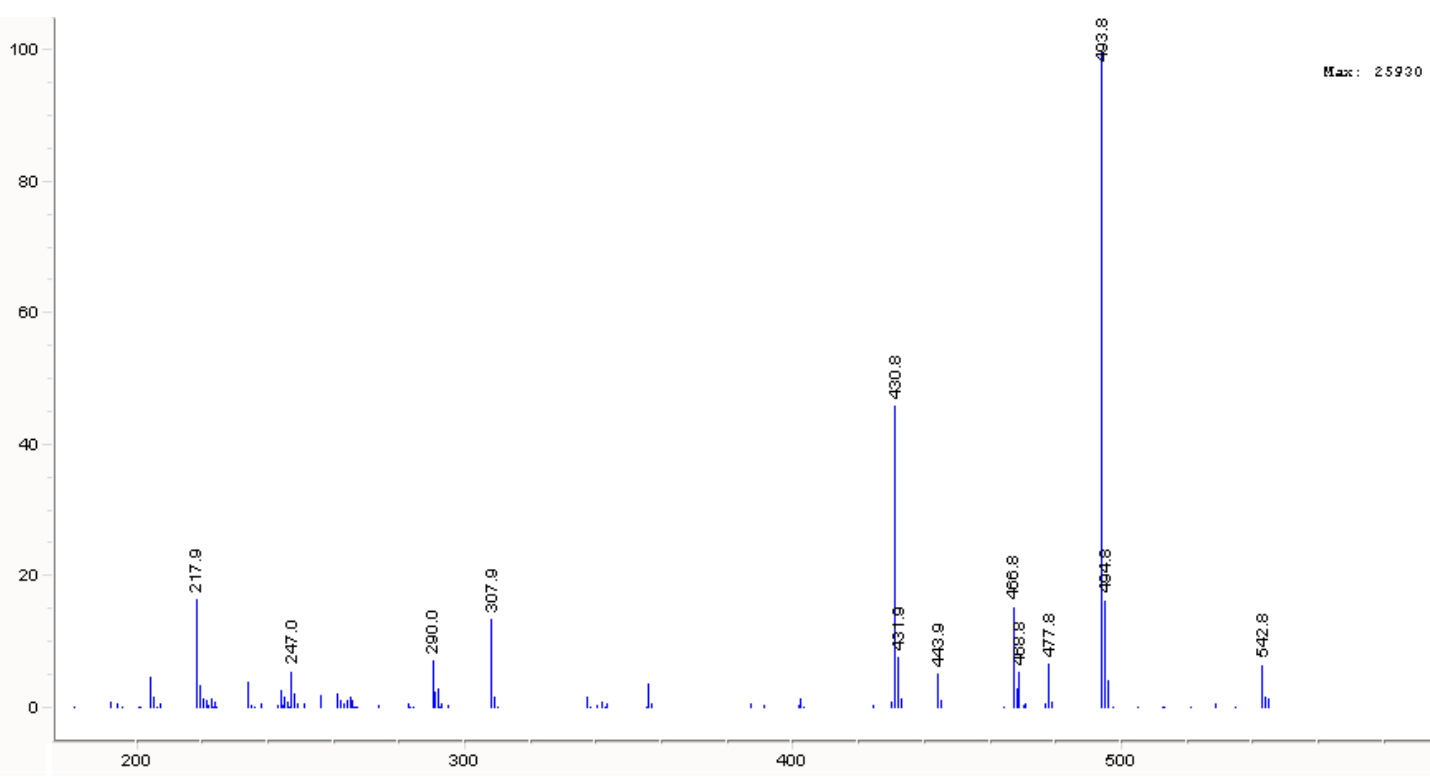

Figure S5. MS spectrum of DATN1

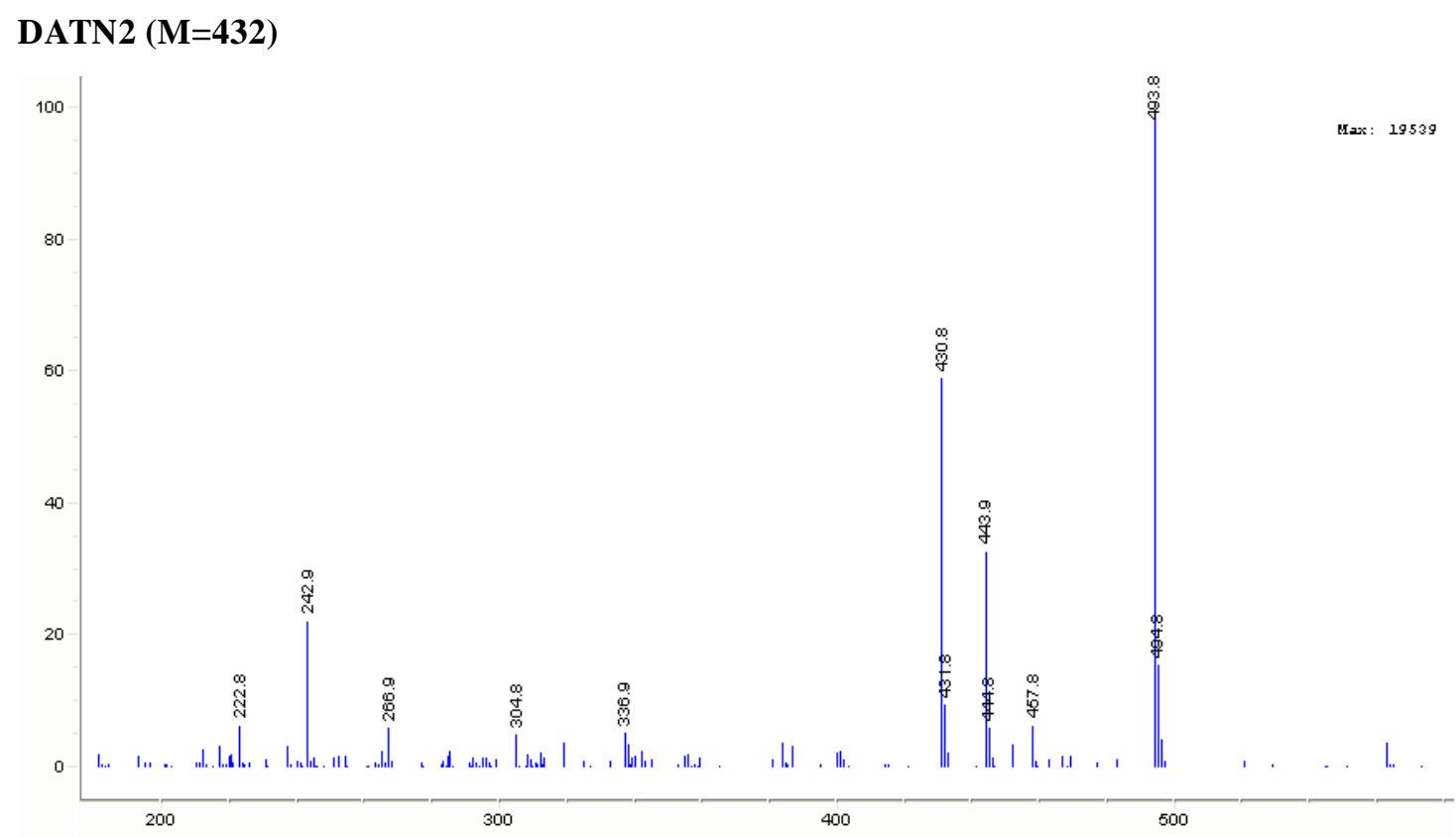

Figure S6. MS spectrum of DATN2 


\section{DATN3 $(M=432)$}

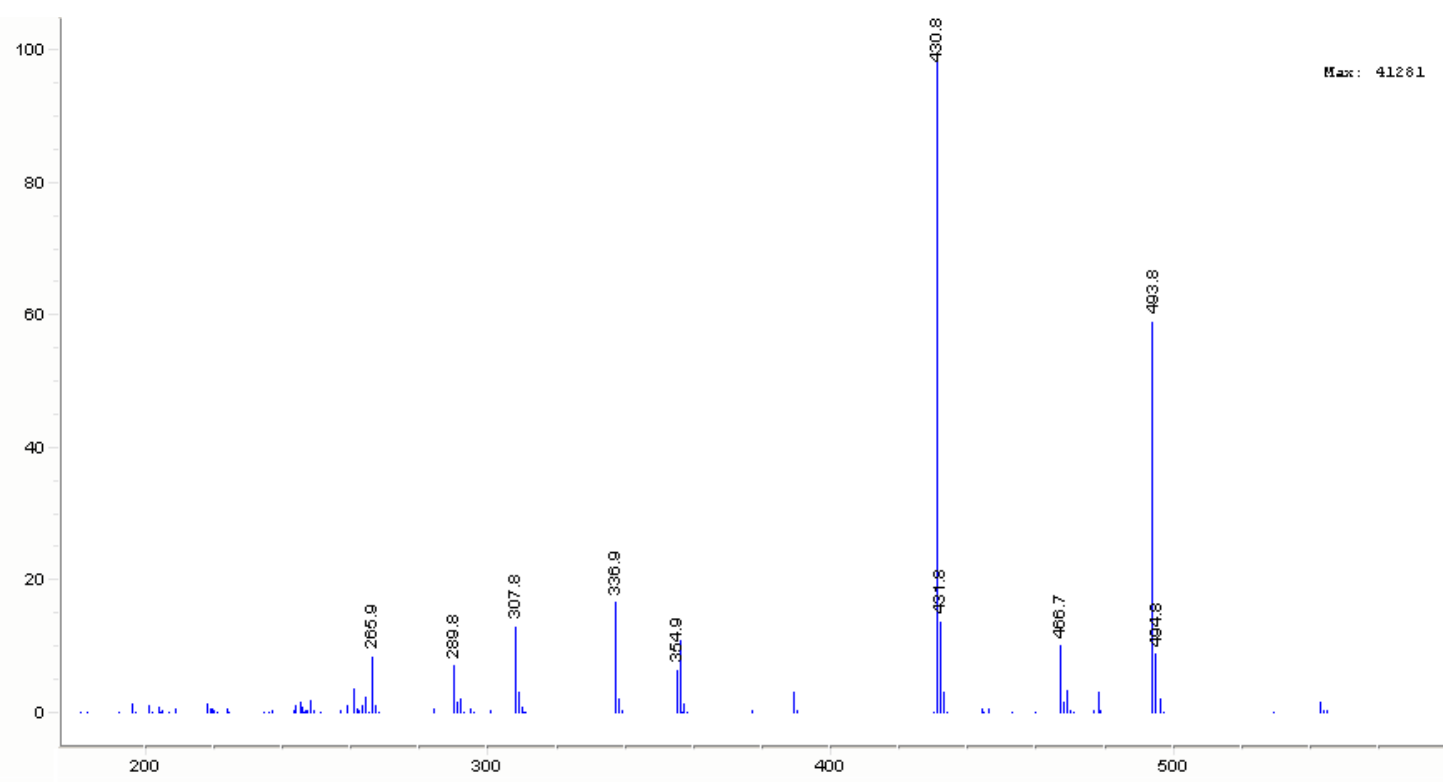

Figure S7. MS spectrum of DATN3

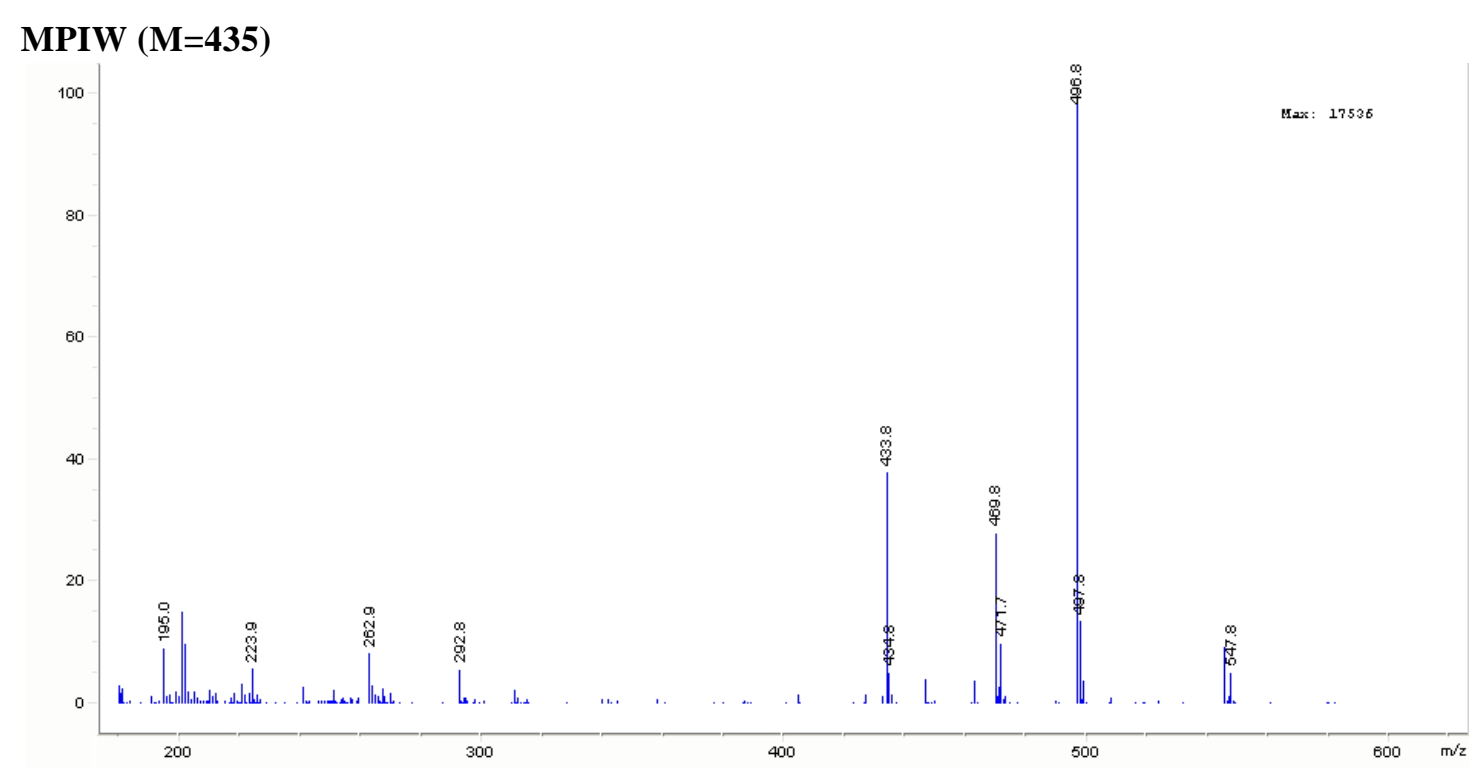

Figure S8. MS spectrum of MPIW 


\section{S4. Quantitative analysis method of reaction components}

The concentrations of reaction components were determined with the method of standard curve. In order to guarantee the accuracy of measurement, the standard curve of every component were made before every analysis process in HPLC, which implied a lot of tedious work to do. Considering the complexity of the experiments, a simple and effective handing method, simultaneously giving consideration to accuracy, was employed. The response factor of a component in HPLC represented the ratio between its concentration and peak area in liquid chromatography, and the ratios $\left(\mathrm{F}_{\mathrm{i}}\right)$ of response factors between HNIW and the nitration intermediates were obtained by experiments in a series of identical concentrations under the same analysis conditions. Thus, a standard curve of HNIW was only required to make before every analysis process, while the other components concentrations could be obtained at the same time.

Table S2. The ratios of response factors between HNIW and nitration intermediates

\begin{tabular}{|c|c|c|c|c|c|c|c|}
\hline $\begin{array}{c}\text { Reaction } \\
\text { Components(i) }\end{array}$ & TADN & TATN & DATN1 & DATN2 & DATN3 & MPIW & HNIW \\
\hline $\mathrm{F}_{\mathrm{i}}$ & 0.30 & 0.39 & 0.51 & 0.51 & 0.59 & 0.71 & 1.00 \\
\hline
\end{tabular}

HNIW : $\quad \mathrm{Y}=\mathrm{ax}+\mathrm{b}$,

$$
\mathrm{x}=(\mathrm{Y}-\mathrm{b}) / \mathrm{a},
$$

$\mathrm{Y}$ and $\mathrm{x}$ are the peak area and concentration of HNIW, respectively.

Intermediate $\mathrm{i}: \quad \mathrm{Y}_{\mathrm{i}} \mathrm{F}_{\mathrm{i}}=\mathrm{Y}$,

$\mathrm{x}_{\mathrm{i}}=\left(\mathrm{Y}_{\mathrm{i}} \mathrm{F}_{\mathrm{i}}-\mathrm{b}\right) / \mathrm{a}$,

$\mathrm{Y}_{\mathrm{i}}$ and $\mathrm{x}_{\mathrm{i}}$ are the peak area and concentration of intermediate $\mathrm{i}$, respectively;

$F_{i}$ is the ratio of response factor between HNIW and intermediate $i$. 
S5. Calculation formula of $R, \mathbf{R}^{2}$ and adjusted $\mathbf{R}^{2}$

SST $($ sum of squares for total $)=\sum\left(y_{i}-y\right)^{2}$

$\operatorname{SSR}($ sum of squares for regression $)=\sum\left(\mathrm{Y}_{\mathrm{i}}-\mathrm{y}\right)^{2}$

$\operatorname{SSE}($ sum of squares for error $)=\sum\left(\mathrm{y}_{\mathrm{i}}-\mathrm{Y}_{\mathrm{i}}\right)^{2}$

$\mathrm{R}^{2}=1-\frac{\mathrm{SSE}}{\mathrm{SST}}$

Adjusted $R^{2}=1-\frac{\operatorname{SSE}(\mathrm{n}-1)}{\operatorname{SST}(\mathrm{n}-\mathrm{k}-1)}$

$\mathrm{y}_{\mathrm{i}}$ and $\mathrm{Y}_{\mathrm{i}}$ represent the experimental data and calculated values, respectively;

$y$ is the mean value of $y_{i}$;

(n-k-1) and (n-1) are the degrees of freedom of SSE and SST, respectively. 